\title{
ON THE GENERAL CLASSIFICATION OF LIE BIALGEBRA STRUCTURES OVER POLYNOMIALS
}

\author{
IULIA POP AND JULIA YERMOLOVA-MAGNUSSON
}

\begin{abstract}
The present paper is a continuation of [5], where Lie bialgebra structures on $\mathfrak{g}[u]$ were studied. These structures fall into different classes labelled by the vertices of the extended Dynkin diagram of $\mathfrak{g}$. In [5] the Lie bialgebras corresponding to $-\alpha_{\max }$ were classified. In the present article, we investigate the Lie bialgebras corresponding to an arbitrary simple root $\alpha$.
\end{abstract}

\section{INTRODUCTION}

Let $\mathfrak{g}$ denote a finite dimensional simple complex Lie algebra. The aim of the present article is to further investigate Lie bialgebra structures on $\mathfrak{g}[u]$ in the framework provided in 3, 5. As it was shown in 3, any Lie bialgebra structure $\delta$ on $\mathfrak{g}[u]$ can be extended to a Lie bialgebra structure $\bar{\delta}$ on $\mathfrak{g}[[u]]$. The classical double associated to any such structure is isomorphic to one of the following Lie algebras:

Case I. $\mathfrak{g}((u))$ together with the following nondegenerate bilinear form

$$
Q_{a(u)}\left(f_{1}(u), f_{2}(u)\right)=\operatorname{Res}_{u=0}\left(a(u) K\left(f_{1}(u), f_{2}(u)\right)\right),
$$

where $K$ is the Killing form of the Lie algebra $\mathfrak{g}((u))$ over $\mathbb{C}((u))$. Here $a(u)=1+\sum_{k=0}^{\infty} a_{k} u^{k}$ is a Taylor series which satisfies certain properties which will be presented later.

Case II. $\mathfrak{g}((u)) \oplus \mathfrak{g}$, endowed with the following nondegenerate bilinear form:

$Q_{a(u)}\left(f_{1}(u)+x_{1}, f_{2}(u)+x_{2}\right)=\operatorname{Res}_{u=0}\left(u^{-1} a(u) K\left(f_{1}(u), f_{2}(u)\right)\right)-K\left(x_{1}, x_{2}\right)$,

for all $f_{1}(u), f_{2}(u) \in \mathfrak{g}((u))$ and $x_{1}, x_{2} \in \mathfrak{g}$. Again $a(u)=1+\sum_{k=0}^{\infty} a_{k} u^{k}$ is a certain Taylor series.

Case III. $\mathfrak{g}((u)) \oplus(\mathfrak{g}+\varepsilon \mathfrak{g})$, where $\varepsilon^{2}=0$. Here we consider

$$
\begin{gathered}
Q_{a(u)}\left(f_{1}(u)+x_{2}+\varepsilon x_{3}, f_{2}(u)+y_{2}+\varepsilon y_{3}\right)=\operatorname{Res}_{u=0}\left(u^{-2} a(u) K\left(f_{1}(u), f_{2}(u)\right)-\right. \\
-K\left(x_{3}, y_{2}\right)-K\left(x_{2}, y_{3}\right),
\end{gathered}
$$

for any $f_{1}(u), f_{2}(u) \in \mathfrak{g}((u))$ and $x_{2}, x_{3}, y_{2}, y_{3} \in \mathfrak{g}$, and $a(u)=1+\sum_{k=0}^{\infty} a_{k} u^{k}$ is a certain Taylor series.

1991 Mathematics Subject Classification. Primary 17B37, 17B62; Secondary 17B81.

Key words and phrases. Lie bialgebra, Lagrangian subalgebra, parabolic subalgebra, admissible triple. 
The Lie bialgebra structure $\bar{\delta}$ (and implicitly $\delta$ ) is uniquely defined by a bounded Lagrangian subalgebra $W$ of $\mathfrak{g}((u)), \mathfrak{g}((u)) \oplus \mathfrak{g}$ or $\mathfrak{g}((u)) \oplus(\mathfrak{g}+\varepsilon \mathfrak{g})$. Moreover, $W$ should be transversal to $\mathfrak{g}[[u]]$.

The classification of bialgebra structures is reduced in this way to the classification of bounded Lagrangian subalgebras $W$ with the above property. Moreover, any such $W$ can be embedded into a special algebra, a so-called maximal order, indexed by vertices of the extended Dynkin diagram of $\mathfrak{g}$. We recall its construction, according to 6]. Let $\mathfrak{h}$ be a Cartan subalgebra of $\mathfrak{g}$ with the corresponding set of roots $R$ and a choice of simple roots $\Gamma$. Denote by $\mathfrak{g}_{\alpha}$ the root space corresponding to a root $\alpha$. Let $\mathfrak{h}(\mathbb{R})=\{h \in \mathfrak{h}: \alpha(h) \in \mathbb{R}, \alpha \in R\}$. Consider the valuation on $\mathbb{C}\left(\left(u^{-1}\right)\right)$ defined by $v\left(\sum_{k \geq n} a_{k} u^{-k}\right)=n$. For any root $\alpha$ and any $h \in \mathfrak{h}(\mathbb{R})$, set $M_{\alpha}(h):=\left\{f \in \mathbb{C}\left(\left(u^{-1}\right)\right): v(f) \geq \alpha(h)\right\}$. Consider

$$
\mathbb{O}_{h}:=\mathfrak{h}\left[\left[u^{-1}\right]\right] \oplus\left(\oplus_{\alpha \in R} M_{\alpha}(h) \otimes \mathfrak{g}_{\alpha}\right) .
$$

Let $\left\{h \in \mathfrak{h}(\mathbb{R}): \alpha(h) \geq 0, \alpha \in \Gamma, \alpha_{\max }(h) \leq 1\right\}$ be the standard simplex. Vertices of the above simplex correspond to vertices of the extended Dynkin diagram of $\mathfrak{g}$, the correspondence being given by the following rule: $0 \leftrightarrow \quad-\alpha_{\max }, h_{i} \leftrightarrow \alpha_{i}$, where $\alpha_{i}\left(h_{j}\right)=\delta_{i j} / k_{j}$, and $k_{j}$ are given by the relation $\sum k_{j} \alpha_{j}=\alpha_{\text {max }}$. One writes $\mathbb{O}_{\alpha}$ instead of $\mathbb{O}_{h}$ if $\alpha$ is the root which corresponds to the vertex $h$, and $\mathbb{O}_{-\alpha_{\max }}$ instead of $\mathbb{O}_{0}$.

According to [3], in case I, $1 / a(u)$ is a polynomial of degree at most 2 , in case II, is at most 1 and in case III, its degree is 0 . Moreover, by means of a change of variable in $\mathbb{C}[u]$ and rescaling the nondegenerate bilinear form $Q_{a(u)}$, one may assume that $a(u)$ has one of the following forms:

(1) $a(u)=1 /\left(1-c_{1} u\right)\left(1-c_{2} u\right)$, for non-zero constants $c_{1} \neq c_{2}$

(2) $a(u)=1 /(1-u)^{2}$

(3) $a(u)=1 / 1-u$

(4) $a(u)=1$.

In what follows we will treat separately case I (subcases 1-4), case II (subcases 3-4 are the only possible), case III (subcase 4 is the only possible).

\section{Lie BIALGEbRA StRUCtURES ON $\mathfrak{g}[u]$ IN CASE I}

Here we consider $\mathfrak{g}((u))$ together with the form

$$
Q_{a(u)}\left(f_{1}(u), f_{2}(u)\right)=\operatorname{Res}_{u=0}\left(a(u) K\left(f_{1}(u), f_{2}(u)\right)\right),
$$

for any $f_{1}(u), f_{2}(u) \in \mathfrak{g}((u))$. In [3] the following result was proved:

Proposition 2.1. [3] There exists a one-to-one correspondence between Lie bialgebra structures $\delta$ on $\mathfrak{g}[u]$ satisfying $D_{\bar{\delta}}(\mathfrak{g}[[u]])=\mathfrak{g}((u))$ and bounded Lagrangian subalgebras $W$ of $\mathfrak{g}((u))$, with respect to the nondegenerate bilinear form $Q_{a(u)}$, and transversal to $\mathfrak{g}[[u]]$.

In 5], we treated this infinite-dimensional problem by first showing that any such $W$ can be embedded into a special algebra, a so-called maximal 
order, indexed by vertices of the extended Dynkin diagram of $\mathfrak{g}$. This embedding allows us to replace our infinite-dimensional problem with a finitedimensional one.

Proposition 2.2. [5] Suppose that $W$ is a bounded Lagrangian subalgebra of $\mathfrak{g}((u))$, with respect to $Q_{a(u)}$ and transversal to $\mathfrak{g}[[u]]$. Then there exists $\sigma \in \operatorname{Aut}_{\mathbb{C}[u]}(\mathfrak{g}[u])$ such that $\sigma(u)(W) \subseteq \mathbb{O}_{\alpha} \cap \mathfrak{g}\left[u, u^{-1}\right]$, where $\alpha$ is either a simple root or $-\alpha_{\max }$.

In [5] the Lie bialgebras corresponding to $-\alpha_{\max }$ were classified. In the present article, we investigate the Lie bialgebras corresponding to an arbitrary simple root $\alpha$.

Consider the Lie algebra $\mathfrak{g} \oplus \mathfrak{g}$, together with the nondegenerate bilinear form

$$
\bar{Q}\left(\left(x_{1}, y_{1}\right),\left(x_{2}, y_{2}\right)\right)=K\left(x_{1}, x_{2}\right)-K\left(y_{1}, y_{2}\right),
$$

for any elements $x_{1}, y_{1}, x_{2}, y_{2} \in \mathfrak{g}$. Let us fix an arbitrary simple root $\alpha$. Denote by $P_{\alpha}^{-}$the standard parabolic subalgebra of $\mathfrak{g}$ spanned by the root spaces of all negative roots and all positive roots which do not contain $\alpha$. Let us denote by $\Delta_{\alpha}$ the set of pairs $(x, y) \in P_{\alpha}^{-} \times P_{\alpha}^{-}$which have the same reductive parts. Then the following result holds:

Theorem 2.3. Let $\alpha$ be a simple root and $k$ its coefficient in the decomposition of $\alpha_{\max }$. Let $a(u)=1 /\left(1-c_{1} u\right)\left(1-c_{2} u\right)$, for non-zero constants $c_{1} \neq c_{2}$.

(i) If $k=1$, there exists a one-to-one correspondence between Lagrangian subalgebras $W$ of $\mathfrak{g}((u))$, with respect to $Q_{a(u)}$, which are transversal to $\mathfrak{g}[[u]]$ and satisfy $W \subseteq \mathbb{O}_{\alpha} \cap \mathfrak{g}\left[u, u^{-1}\right]$, and Lagrangian subalgebras in $\mathfrak{g} \oplus \mathfrak{g}$, with respect to $\bar{Q}$, transversal to $\Delta_{\alpha}$.

(ii) If $k>1$ then there are no Lagrangian subalgebras $W$ of $\mathfrak{g}((u))$, with respect to $Q_{a(u)}$, which are transversal to $\mathfrak{g}[[u]]$ and satisfy $W \subseteq \mathbb{O}_{\alpha} \cap \mathfrak{g}\left[u, u^{-1}\right]$.

Proof. Part (ii) was proved in [3]. We will prove (i). Assume that $\alpha$ has coefficient 1 in $\alpha_{\max }$. Then the corresponding maximal order $\mathbb{O}_{\alpha}$ is given by the formula

$$
\mathbb{O}_{\alpha}=u^{-1} \mathfrak{g}_{1}\left[\left[u^{-1}\right]\right]+\mathfrak{g}_{0}\left[\left[u^{-1}\right]\right]+u \mathfrak{g}_{-1}\left[\left[u^{-1}\right]\right],
$$

where $\mathfrak{g}_{1}$ is the sum of the root spaces of positive roots which contain $\alpha$ (with coefficient 1), $\mathfrak{g}_{-1}$ is the sum of the root spaces of negative roots which contain $\alpha$ (with coefficient 1 ), and $\mathfrak{g}_{0}$ consists of $\mathfrak{h}$ and the root spaces of all roots which do not contain $\alpha$.

Then $\mathbb{O}_{\alpha} \cap \mathfrak{g}\left[u, u^{-1}\right]=u^{-1} \mathfrak{g}_{1}\left[u^{-1}\right]+\mathfrak{g}_{0}\left[u^{-1}\right]+u \mathfrak{g}_{-1}\left[u^{-1}\right]$ and $\left(\mathbb{O}_{\alpha} \cap\right.$ $\left.\mathfrak{g}\left[u, u^{-1}\right]\right)^{\perp}=\left(u^{-1}-c_{1}\right)\left(u^{-1}-c_{2}\right)\left(u^{-1} \mathfrak{g}_{1}\left[u^{-1}\right]+\mathfrak{g}_{0}\left[u^{-1}\right]+u \mathfrak{g}_{-1}\left[u^{-1}\right]\right)$.

Let us construct an isomorphism $\bar{\phi}$ between $\frac{\mathbb{O}_{\alpha} \cap \mathfrak{g}\left[u, u^{-1}\right]}{\left(\mathbb{O}_{\alpha} \cap \mathfrak{g}\left[u, u^{-1}\right]\right)^{\perp}}$ and $\mathfrak{g} \oplus \mathfrak{g}$. We consider $\phi: \mathbb{O}_{\alpha} \cap \mathfrak{g}\left[u, u^{-1}\right] \longrightarrow \mathfrak{g} \oplus \mathfrak{g}$ given by $\phi\left(u^{-1} p_{1}\left(u^{-1}\right)+p_{0}\left(u^{-1}\right)+\right.$ $\left.u p_{-1}\left(u^{-1}\right)\right)=\left(c_{1} p_{1}\left(c_{1}\right)+p_{0}\left(c_{1}\right)+c_{1}^{-1} p_{-1}\left(c_{1}\right), c_{2} p_{1}\left(c_{2}\right)+p_{0}\left(c_{2}\right)+c_{2}^{-1} p_{-1}\left(c_{2}\right)\right)$, where $p_{1} \in \mathfrak{g}_{1}\left[u^{-1}\right], p_{0} \in \mathfrak{g}_{0}\left[u^{-1}\right]$ and $p_{-1} \in \mathfrak{g}_{-1}\left[u^{-1}\right]$. One can easily check 
that the kernel of this map is exactly $\left(\mathbb{O}_{\alpha} \cap \mathfrak{g}\left[u, u^{-1}\right]\right)^{\perp}$. Moreover, $\phi$ is surjective. Indeed, for any elements $a, b$ of $\mathfrak{g}$, let us uniquely decompose $a=a_{1}+a_{0}+a_{-1}, b=b_{1}+b_{0}+b_{-1}$ with $a_{1}, b_{1} \in \mathfrak{g}_{1}, a_{0}, b_{0} \in \mathfrak{g}_{0}, a_{-1}, b_{-1} \in$ $\mathfrak{g}_{-1}$. Then one can find first degree polynomials $p_{1} \in \mathfrak{g}_{1}\left[u^{-1}\right], p_{0} \in \mathfrak{g}_{0}\left[u^{-1}\right]$ and $p_{-1} \in \mathfrak{g}_{-1}\left[u^{-1}\right]$ such that $c_{1} p_{1}\left(c_{1}\right)=a_{1}, c_{2} p_{1}\left(c_{2}\right)=b_{1} ; p_{0}\left(c_{1}\right)=a_{0}$, $p_{0}\left(c_{2}\right)=b_{0} ; c_{1}^{-1} p_{-1}\left(c_{1}\right)=a_{-1}, c_{2}^{-1} p_{-1}\left(c_{2}\right)=b_{-1}$.

Thus one obtains an isomorphism $\bar{\phi}: \frac{\mathbb{O}_{\alpha} \cap \mathfrak{g}\left[u, u^{-1}\right]}{\left(\mathbb{O}_{\alpha} \cap \mathfrak{g}\left[u, u^{-1}\right]\right)^{\perp}} \longrightarrow \mathfrak{g} \oplus \mathfrak{g}$. This implies that we have a 1-1 correspondence between Lagrangian subalgebras $W$ contained in $\mathbb{O}_{\alpha} \cap \mathfrak{g}\left[u, u^{-1}\right]$ and Lagrangian subalgebras $\bar{W}=\phi(W)$ in $\mathfrak{g} \oplus \mathfrak{g}$. Moreover $W$ is transversal to $\mathfrak{g}[[u]]$ if and only if $\bar{W}$ is transversal to $\phi\left(\mathbb{O}_{\alpha} \cap \mathfrak{g}[u]\right)$. On the other hand, $\phi\left(\mathbb{O}_{\alpha} \cap \mathfrak{g}[u]\right)$ consists of all pairs of the form $\left(a_{0}+c_{1}^{-1} b_{0}+b_{1}, a_{0}+c_{2}^{-1} b_{0}+b_{1}\right)$, where $a_{0} \in \mathfrak{g}_{0}, b_{0}, b_{1} \in \mathfrak{g}_{-1}$, which is precisely $\Delta_{\alpha}$. We note that $\mathfrak{g}_{0}$ is the reductive part of $P_{\alpha}^{-}=\mathfrak{g}_{0}+\mathfrak{g}_{-1}$. The proof is complete.

Remark 2.4. The classification of all Lagrangian subalgebras $\bar{W}$ of $\mathfrak{g} \oplus \mathfrak{g}$ transversal to $\Delta_{\alpha}$ was accomplished in [2], Theorem 11. This result states that, up to a conjugation which preserves $\Delta_{\alpha}$, a subalgebra $\bar{W}$ can be obtained from a pair formed by a triple $\left(\Gamma_{1}, \Gamma_{2}, A\right)$ and a tensor $r \in \mathfrak{h} \otimes \mathfrak{h}$ such that $\left(\Gamma_{1}, \Gamma_{2}, A\right)$ is of type I or II and $r$ satisfies certain equations depending of the case. We recall that $\Gamma_{1}, \Gamma_{2}$ are subsets of $\Gamma$ and $A$ is an isometry between them. A triple is of type I if $\alpha \notin \Gamma_{2}$ and $\left(\Gamma_{1}, \theta\left(\Gamma_{2}\right), \theta(A)\right)$ is admissible in the sense of Belavin-Drinfeld (see [1]). A triple is of type II if $\alpha \in \Gamma_{2}, A(\beta)=\alpha$ and $\left(\Gamma_{1} \backslash\{\beta\}, \theta\left(\Gamma_{2} \backslash\{\alpha\}\right), \theta(A)\right)$ is admissible in the sense of Belavin-Drinfeld. Here $\theta$ denotes the Cartan involution of $\mathfrak{g}_{0}$.

Remark 2.5. The classification of Lagrangian subalgebras $\bar{W}$ can also be given using triples of the form $\left(\Gamma_{1}, \Gamma_{2}, A\right)$, where $\Gamma_{1} \subseteq \Gamma^{e x t} \backslash\{\alpha\}, \Gamma_{2} \subseteq \Gamma$ and $A: \Gamma_{1} \longrightarrow \Gamma_{2}$ is an isometry. In this presentation, the case $k=1$ can be considered a particular case of the classification result which will be given in Remark 2.9.

Consider the Lie algebra $\mathfrak{g}[\varepsilon]$ with $\varepsilon^{2}=0$, endowed with the following invariant form:

$$
\bar{Q}_{\varepsilon}\left(x_{1}+\varepsilon x_{2}, y_{1}+\varepsilon y_{2}\right)=K\left(x_{1}, y_{2}\right)+K\left(x_{2}, y_{1}\right),
$$

for any elements $x_{1}, y_{1}, x_{2}, y_{2}$ of $\mathfrak{g}$.

Theorem 2.6. Let $\alpha$ be a simple root and $k$ its coefficient in the decomposition of $\alpha_{\max }$. Let $a(u)=\frac{1}{(1-u)^{2}}$.

(i) If $k=1$, there exists a one-to-one correspondence between Lagrangian subalgebras $W$ of $\mathfrak{g}((u))$, with respect to $Q_{a(u)}$, which are transversal to $\mathfrak{g}[[u]]$ and satisfy $W \subseteq \mathbb{O}_{\alpha} \cap \mathfrak{g}\left[u, u^{-1}\right]$, and Lagrangian subalgebras in $\mathfrak{g}[\varepsilon]$, with respect to $\bar{Q}_{\varepsilon}$, transversal to $P_{\alpha}^{-}+\varepsilon\left(P_{\alpha}^{-}\right)^{\perp}$.

(ii) If $k>1$ then there are no Lagrangian subalgebras $W$ of $\mathfrak{g}((u))$, with respect to $Q_{a(u)}$, which are transversal to $\mathfrak{g}[[u]]$ and satisfy $W \subseteq \mathbb{O}_{\alpha} \cap \mathfrak{g}\left[u, u^{-1}\right]$. 
Proof. We will only prove (i) (for (ii) see [3]). Because $k=1$, one has the following: $\mathbb{O}_{\alpha} \cap \mathfrak{g}\left[u, u^{-1}\right]=u^{-1} \mathfrak{g}_{1}\left[u^{-1}\right]+\mathfrak{g}_{0}\left[u^{-1}\right]+u \mathfrak{g}_{-1}\left[u^{-1}\right]$ and $\left(\mathbb{O}_{\alpha} \cap\right.$ $\left.\mathfrak{g}\left[u, u^{-1}\right]\right)^{\perp}=\left(u^{-1}-1\right)^{2}\left(u^{-1} \mathfrak{g}_{1}\left[u^{-1}\right]+\mathfrak{g}_{0}\left[u^{-1}\right]+u \mathfrak{g}_{-1}\left[u^{-1}\right]\right)$.

Let us construct an isomorphism $\bar{\phi}$ between $\frac{\mathbb{O}_{\alpha} \cap \mathfrak{g}\left[u, u^{-1}\right]}{\left(\mathbb{O}_{\alpha} \cap \mathfrak{g}\left[u, u^{-1}\right]\right)^{\perp}}$ and $\mathfrak{g}[\varepsilon]$.

We consider $\phi: \mathbb{O}_{\alpha} \cap \mathfrak{g}\left[u, u^{-1}\right] \longrightarrow \mathfrak{g}[\varepsilon]$ given by $\phi\left(u^{-1} p_{1}\left(u^{-1}\right)+p_{0}\left(u^{-1}\right)+\right.$ $\left.u p_{-1}\left(u^{-1}\right)\right)=(1+\varepsilon) p_{1}(1+\varepsilon)+p_{0}(1+\varepsilon)+(1-\varepsilon) p_{-1}(1+\varepsilon)$, where $p_{1} \in \mathfrak{g}_{1}\left[u^{-1}\right]$, $p_{0} \in \mathfrak{g}_{0}\left[u^{-1}\right]$ and $p_{-1} \in \mathfrak{g}_{-1}\left[u^{-1}\right]$. One can check that the kernel of this map is the ideal generated by $\left(u^{-1}-1\right)^{2}$. The map $\phi$ is also surjective. Indeed, let us write $a+\varepsilon b=\left(a_{1}+a_{0}+a_{-1}\right)+\varepsilon\left(b_{1}+b_{0}+b_{-1}\right)$. One can uniquely find first degree polynomials $p_{1}, p_{0}$ and $p_{-1}$ such that $\phi\left(u^{-1} p_{1}\left(u^{-1}\right)+p_{0}\left(u^{-1}\right)+\right.$ $\left.u p_{-1}\left(u^{-1}\right)\right)=a+\varepsilon b$. Straightforward computations give: $p_{1}\left(u^{-1}\right)=\left(2 a_{1}-\right.$ $\left.b_{1}\right)+u^{-1}\left(b_{1}-a_{1}\right), p_{0}\left(u^{-1}\right)=a_{0}-b_{0}+u^{-1} b_{0}, p_{-1}\left(U^{-1}\right)=-b_{-1}+u^{-1}\left(a_{-1}+\right.$ $\left.b_{-1}\right)$.

By means of the above isomorphism, we have a 1-1 correspondence between Lagrangian subalgebras $W$ of $\mathfrak{g}((u))$, with respect to $Q_{a(u)}$, which are transversal to $\mathfrak{g}[[u]]$ and satisfy $W \subseteq \mathbb{O}_{\alpha} \cap \mathfrak{g}\left[u, u^{-1}\right]$, and Lagrangian subalgebras in $\mathfrak{g}[\varepsilon]$, with respect to $\bar{Q}_{\varepsilon}$, transversal to $\phi\left(\mathbb{O}_{\alpha} \cap \mathfrak{g}[u]\right)$.

On the other hand, $\phi\left(\mathbb{O}_{\alpha} \cap \mathfrak{g}[u]\right)=\phi\left(\mathfrak{g}_{0}+u\left(\mathfrak{g}_{-1}+u^{-1} \mathfrak{g}_{-1}\right)\right.$ which consists of elements of the form $a_{0}+(1-\varepsilon) b_{-1}+c_{-1}$, for all $a_{0} \in \mathfrak{g}_{0}$ and $b_{-1}, c_{-1} \in \mathfrak{g}_{-1}$. Since, $P_{\alpha}^{-}=\mathfrak{g}_{0}+\mathfrak{g}_{-1}$, it follows that $\phi\left(\mathbb{O}_{\alpha} \cap \mathfrak{g}[u]\right)$ is precisely $P_{\alpha}^{-}+\varepsilon\left(P_{\alpha}^{-}\right)^{\perp}$. This ends the proof.

Remark 2.7. In [6] it was shown that Lagrangian subalgebras of $\mathfrak{g}[\varepsilon]$, with respect to $\bar{Q}_{\varepsilon}$, and transversal to $P_{\alpha}^{-}+\varepsilon\left(P_{\alpha}^{-}\right)^{\perp}$ are in a 1-1 correspondence with pairs $(L, B)$, where $L$ is a subalgebra of $\mathfrak{g}$ satisfying $L+P_{\alpha}^{-}=\mathfrak{g}$ and $B$ is a 2-cocycle on $L$ nondegenerate on $L \cap P_{\alpha}^{-}$.

Let us assume again that $\alpha$ is a simple root with coefficient $k$ in $\alpha_{\max }$. Denote by $L_{\alpha}$ the Lie subalgebra of $\mathfrak{g}$ whose Dynkin diagram is obtained from the extended Dynkin diagram of $\mathfrak{g}$ by erasing $\alpha$. Then the restriction of the bilinear form $\bar{Q}$ on $\mathfrak{g} \oplus \mathfrak{g}$ is nondegenerate on $L_{\alpha} \times \mathfrak{g}$.

Consider the standard parabolic subalgebra of $L_{\alpha}$ corresponding to $-\alpha_{\max }$, $P_{\alpha_{\max }}^{+}$. We note that $P_{\alpha_{\max }}^{+}$and $P_{\alpha}^{-}$have equal reductive components. Let $\Delta_{\alpha, \alpha_{\max }}$ be the set of pairs $(x, y) \in P_{\alpha_{\max }}^{+} \times P_{\alpha}^{-}$with equal reductive parts.

Theorem 2.8. Let $\alpha$ be a simple root and $k$ its coefficient in the decomposition of $\alpha_{\max }$. Let $a(u)=\frac{1}{1-u}$.

There exists a one-to-one correspondence between Lagrangian subalgebras $W$ of $\mathfrak{g}((u))$, with respect to $Q_{a(u)}$, which are transversal to $\mathfrak{g}[[u]]$ and satisfy $W \subseteq \mathbb{O}_{\alpha} \cap \mathfrak{g}\left[u, u^{-1}\right]$, and Lagrangian subalgebras in $L_{\alpha} \oplus \mathfrak{g}$, with respect to $\bar{Q}$, transversal to $\Delta_{\alpha, \alpha_{\max }}$.

Proof. For each $r,-k \leq r \leq k$, let $R_{r}$ denote the set of all roots which contain $\alpha$ with coefficient $r$. Let $\mathfrak{g}_{0}=\mathfrak{h} \oplus \sum_{\beta \in R_{0}} \mathfrak{g}_{\beta}$ and $\mathfrak{g}_{r}=\sum_{\beta \in R_{r}} \mathfrak{g}_{\beta}$. 
Then

$$
\begin{gathered}
\mathbb{O}_{\alpha}=\sum_{r=1}^{k} u^{-1} \mathfrak{g}_{r}\left[\left[u^{-1}\right]\right]+\sum_{r=1-k}^{0} \mathfrak{g}_{r}\left[\left[u^{-1}\right]\right]+u \mathfrak{g}_{-k}\left[\left[u^{-1}\right]\right], \\
\mathbb{O}_{\alpha} \cap \mathfrak{g}\left[u, u^{-1}\right]=\sum_{r=1}^{k} u^{-1} \mathfrak{g}_{r}\left[u^{-1}\right]+\sum_{r=1-k}^{0} \mathfrak{g}_{r}\left[u^{-1}\right]+u \mathfrak{g}_{-k}\left[u^{-1}\right], \\
\left(\mathbb{O}_{\alpha} \cap \mathfrak{g}\left[u, u^{-1}\right]\right)^{\perp}=(1-u)\left(u^{-3} \mathfrak{g}_{k}\left[u^{-1}\right]+\sum_{r=0}^{k-1} u^{-2} \mathfrak{g}_{r}\left[u^{-1}\right]+\sum_{r=-k}^{-1} u^{-1} \mathfrak{g}_{r}\left[u^{-1}\right]\right) .
\end{gathered}
$$

Let $\phi: \mathbb{O}_{\alpha} \cap \mathfrak{g}\left[u, u^{-1}\right] \longrightarrow\left(\mathfrak{g}_{k}+\mathfrak{g}_{0}+\mathfrak{g}_{-k}\right) \oplus \mathfrak{g}$ be defined by

$$
\begin{gathered}
\phi\left(\sum_{r=1}^{k} u^{-1} p_{r}\left(u^{-1}\right)+\sum_{r=1-k}^{0} p_{r}\left(u^{-1}\right)+u p_{-k}\left(u^{-1}\right)\right)= \\
\left(p_{k}(0)+p_{0}(0)+p_{-k}(0), \sum_{r=1}^{k} p_{r}(1)+\sum_{r=1-k}^{0} p_{r}(1)+p_{-k}(1)\right) .
\end{gathered}
$$

One can check that $\phi$ is an epimorphism whose kernel is $\left.\mathbb{O}_{\alpha} \cap \mathfrak{g}\left[u, u^{-1}\right]\right)^{\perp}$. By means of this morphism, we have a 1-1 correspondence between Lagrangian subalgebras $W$ of $\mathfrak{g}((u))$, contained in $\mathbb{O}_{\alpha} \cap \mathfrak{g}\left[u, u^{-1}\right]$, and Lagrangian subalgebras $\bar{W}$ of $\left(\mathfrak{g}_{k}+\mathfrak{g}_{0}+\mathfrak{g}_{-k}\right) \oplus \mathfrak{g}$. We observe that $\mathfrak{g}_{k}+\mathfrak{g}_{0}+\mathfrak{g}_{-k}=L_{\alpha}$. On the other hand, $\mathbb{O}_{\alpha} \cap \mathfrak{g}[u]=\sum_{r=1-k}^{0} \mathfrak{g}_{r}+u\left(\mathfrak{g}_{-k}+u^{-1} \mathfrak{g}_{-k}\right)$. Its image in $L_{\alpha} \oplus \mathfrak{g}$ via $\phi$ consists of elements of the form $\left(a_{0}+a_{-k}, a_{0}+\sum_{r=-k}^{-1} a_{r}+b_{-k}\right)$, where $a_{r} \in \mathfrak{g}_{r}, a_{-k}, b_{-k} \in \mathfrak{g}_{-k}$. Since $P_{\alpha_{\max }}^{+}=\mathfrak{g}_{0}+\mathfrak{g}_{-k}$ and $P_{\alpha}^{-}=\mathfrak{g}_{0}+\mathfrak{g}_{1}+\ldots+\mathfrak{g}_{-k}$, this set coincides with $\Delta_{\alpha, \alpha_{\max }}$. The proof is now complete.

Remark 2.9. The classification of all Lagrangian subalgebras $\bar{W}$ of $\mathfrak{g} \oplus \mathfrak{g}$ transversal to $\Delta_{\alpha, \alpha_{\max }}$ was accomplished in [4], Theorem 2.13. This result states that, up to a conjugation which preserves $\Delta_{\alpha, \alpha_{\max }}$, a subalgebra $\bar{W}$ can be obtained from a pair formed by a triple $\left(\Gamma_{1}, \Gamma_{2}, A\right)$ of type I or II and a subspace $\mathfrak{i}_{\mathfrak{a}}$ satisfying a certain condition. Let us recall how types $\mathrm{I}$ and II are defined. Let $i$ denote the embedding of $\mathfrak{g}_{0}$ into $L_{\alpha}$. Here one considers triples of the form $\left(\Gamma_{1}, \Gamma_{2}, A\right)$, where $\Gamma_{1} \subseteq \Gamma^{e x t} \backslash\{\alpha\}, \Gamma_{2} \subseteq \Gamma$ and $A$ is an isometry between $\Gamma_{1}$ and $\Gamma_{2}$. A triple $\left(\Gamma_{1}, \Gamma_{2}, A\right)$ is called of type $I$ if $\alpha \notin \Gamma_{2}$ and $\left(\Gamma_{1}, i\left(\Gamma_{2}\right), i A\right)$ is an admissible triple in the sense of BelavinDrinfeld. The triple $\left(\Gamma_{1}, \Gamma_{2}, A\right)$ is called of type $I I$ if $\alpha \in \Gamma_{2}$ and $A(\beta)=\alpha$, for some $\beta \in \Gamma_{1}$ and $\left(\Gamma_{1} \backslash\{\beta\}, i\left(\Gamma_{2}^{\prime} \backslash\{\alpha\}\right), i A^{\prime}\right)$ is an admissible triple in the sense of Belavin-Drinfeld. The space $\mathfrak{i}_{\mathfrak{a}}$ is a Lagrangian subspace of $\mathfrak{a}:=\left\{\left(h_{1}, h_{2}\right) \in \mathfrak{h} \times \mathfrak{h}: \beta\left(h_{1}\right)=0, \gamma\left(h_{2}\right)=0, \forall \beta \in \Gamma_{1}, \forall \gamma \in \Gamma_{2}\right\}$.

Finally, the remaining case $a(u)=1$ follows by reformulating Prop. 3.2.1. from [6]. The result is the following:

Let $\alpha$ be a simple root with coefficient $k$. There exists a $1-1$ correspondence between Lagrangian subalgebras $W$ of $\mathfrak{g}((u))$, with respect to $Q_{a(u)}$, 
which are transversal to $\mathfrak{g}[[u]]$ and satisfy $W \subseteq \mathbb{O}_{\alpha} \cap \mathfrak{g}\left[u, u^{-1}\right]$, and Lagrangian subalgebras $\bar{W}$ in $\left(L_{\alpha}+\varepsilon^{k} L_{\alpha}\right) \oplus\left(\oplus_{r} \varepsilon^{r} V_{\alpha, r}\right)$ transversal to $\left(P_{\alpha}^{-}+\right.$ $\left.\varepsilon^{k}\left(P_{\alpha}^{-}\right)^{\perp}\right) \oplus\left(\oplus_{r} \varepsilon^{r} P_{\alpha, r}^{-}\right)$(see notation in [6], p. 537).

This concludes the analysis of the Lie bialgebra structures in case I.

\section{Lie Bialgebra StRuCtures on $\mathfrak{g}[u]$ In CASE II}

Consider $\mathfrak{g}((u)) \oplus \mathfrak{g}$ endowed with

$$
\begin{gathered}
Q_{a(u)}\left(f_{1}(u)+x_{1}, f_{2}(u)+x_{2}\right)=\operatorname{Res}_{u=0}\left(u^{-1} a(u) K\left(f_{1}(u), f_{2}(u)\right)\right) \\
-K\left(x_{1}, x_{2}\right)
\end{gathered}
$$

for all $f_{1}(u), f_{2}(u) \in \mathfrak{g}((u))$ and $x_{1}, x_{2} \in \mathfrak{g}$. According to [3], the following statement holds:

Proposition 3.1. There exists a one-to-one correspondence between Lie bialgebra structures $\delta$ on $\mathfrak{g}[u]$ satisfying $D_{\bar{\delta}}(\mathfrak{g}[[u]])=\mathfrak{g}((u)) \oplus \mathfrak{g}$ and bounded Lagrangian subalgebras $W$ of $\mathfrak{g}((u)) \oplus \mathfrak{g}$, with respect to the nondegenerate bilinear form $Q_{a(u)}$, and transversal to $\mathfrak{g}[[u]]$.

For any $\sigma \in \operatorname{Aut}_{\mathbb{C}[u]}(\mathfrak{g}[u])$, denote by $\tilde{\sigma}(u)=\sigma(u) \oplus \sigma(0)$, regarded as an automorphism of $\mathfrak{g}((u)) \oplus \mathfrak{g}$. According to [5], the following result holds:

Proposition 3.2. Suppose that $W$ is a bounded Lagrangian subalgebra of $\mathfrak{g}((u)) \oplus \mathfrak{g}$, with respect to $Q_{a(u)}$ and transversal to $\mathfrak{g}[[u]]$. Then there exists $\sigma \in \operatorname{Aut}_{\mathbb{C}[u]}(\mathfrak{g}[u])$ such that $\tilde{\sigma}(u)(W) \subseteq\left(\mathbb{O}_{\alpha} \cap \mathfrak{g}\left[u, u^{-1}\right]\right) \oplus \mathfrak{g}$, where $\alpha$ is either a simple root or $-\alpha_{\max }$.

Theorem 3.3. Let $\alpha$ be a simple root and $k$ its coefficient in the decomposition of $\alpha_{\max }$. Let $a(u)=\frac{1}{1-u}$.

(i) If $k=1$, there exists a one-to-one correspondence between Lagrangian subalgebras $W$ of $\mathfrak{g}((u)) \oplus \mathfrak{g}$, with respect to $Q_{a(u)}$, which are transversal to $\mathfrak{g}[[u]]$ and satisfy $W \subseteq\left(\mathbb{O}_{\alpha} \cap \mathfrak{g}\left[u, u^{-1}\right]\right) \oplus \mathfrak{g}$, and Lagrangian subalgebras in $\mathfrak{g} \oplus \mathfrak{g}$, with respect to $\bar{Q}$, transversal to $\Delta_{\alpha}$.

(ii) If $k>1$, there are no Lagrangian subalgebras $W$ of $\mathfrak{g}((u)) \oplus \mathfrak{g}$, with respect to $Q_{a(u)}$, which are transversal to $\mathfrak{g}[[u]]$ and satisfy $W \subseteq\left(\mathbb{O}_{\alpha} \cap\right.$ $\left.\mathfrak{g}\left[u, u^{-1}\right]\right) \oplus \mathfrak{g}$.

Proof. If $k=1$, then $\mathbb{O}_{\alpha} \cap \mathfrak{g}\left[u, u^{-1}\right]=u^{-1} \mathfrak{g}_{1}\left[u^{-1}\right]+\mathfrak{g}_{0}\left[u^{-1}\right]+u \mathfrak{g}_{-1}\left[u^{-1}\right]$ and $\left(\mathbb{O}_{\alpha} \cap \mathfrak{g}\left[u, u^{-1}\right] \oplus \mathfrak{g}\right)^{\perp}=\left(u^{-1}-1\right)\left(u^{-1} \mathfrak{g}_{1}\left[u^{-1}\right]+\mathfrak{g}_{0}\left[u^{-1}\right]+u \mathfrak{g}_{-1}\left[u^{-1}\right]\right)$. Then there exists an epimorphism

$$
\phi: \mathbb{O}_{\alpha} \cap \mathfrak{g}\left[u, u^{-1}\right] \oplus \mathfrak{g} \longrightarrow \mathfrak{g} \oplus \mathfrak{g}
$$

given by

$$
\phi\left(\left(u^{-1} p_{1}\left(u^{-1}\right)+p_{0}\left(u^{-1}\right)+u p_{-1}\left(u^{-1}\right), a\right)=p_{1}(1)+p_{0}(1)+p_{-1}(1), a\right),
$$

for all $p_{1}\left(u^{-1}\right) \in \mathfrak{g}_{1}\left[u^{-1}\right], p_{0}\left(u^{-1}\right) \in \mathfrak{g}_{0}\left[u^{-1}\right], p_{-1}\left(u^{-1}\right) \in \mathfrak{g}_{-1}\left[u^{-1}\right]$ and $a \in \mathfrak{g}$. Obviously the kernel of $\phi$ is $\left(\mathbb{O}_{\alpha} \cap \mathfrak{g}\left[u, u^{-1}\right] \oplus \mathfrak{g}\right)^{\perp}$. Let us denote by $\bar{\phi}$ the isomorphism induced by $\phi$ between the quotient and $\mathfrak{g} \oplus \mathfrak{g}$. One can easily 
check that $\bar{\phi}\left(\left(\mathbb{O}_{\alpha} \oplus \mathfrak{g}\right) \cap \mathfrak{g}[u]\right)=\Delta_{\alpha}$ and thus we have a correspondence between $W$ and Lagrangian subalgebras $\bar{W}$ of $\mathfrak{g} \oplus \mathfrak{g}$ transversal to $\Delta_{\alpha}$.

Statement (ii) was proved in [3]. This ends the proof.

Remark 3.4. We see that there is an analogy between the above result and Theorem 2.3. Therefore Lagrangian subalgebras $W$ in $\mathfrak{g}((u)) \oplus \mathfrak{g}$ with the required properties can be expressed using the data given in Remark 2.4 and 2.5 .

The remaining case to be discussed is $a(u)=1$.

Theorem 3.5. Suppose $a(u)=1$ and let $\alpha$ be a simple root and $k$ its coefficient in the decomposition of $\alpha_{\max }$. Then there exists a one-to-one correspondence between Lagrangian subalgebras $W$ of $\mathfrak{g}((u)) \oplus \mathfrak{g}$, with respect to $Q_{a(u)}$, which are transversal to $\mathfrak{g}[[u]]$ and satisfy $W \subseteq\left(\mathbb{O}_{\alpha} \cap \mathfrak{g}\left[u, u^{-1}\right]\right) \oplus \mathfrak{g}$ and Lagrangian subalgebras in $L_{\alpha} \oplus \mathfrak{g}$, with respect to $\bar{Q}$, transversal to $\Delta_{\alpha, \alpha_{\max }}$.

Proof. We will use the same notation as in the proof of Theorem 2.8. We recall the following:

$$
\begin{gathered}
\mathbb{O}_{\alpha} \cap \mathfrak{g}\left[u, u^{-1}\right]=\sum_{r=1}^{k} u^{-1} \mathfrak{g}_{r}\left[u^{-1}\right]+\sum_{r=1-k}^{0} \mathfrak{g}_{r}\left[u^{-1}\right]+u \mathfrak{g}_{-k}\left[u^{-1}\right] \\
\left(\mathbb{O}_{\alpha} \cap \mathfrak{g}\left[u, u^{-1}\right] \oplus \mathfrak{g}\right)^{\perp}=\sum_{-k}^{-1} \mathfrak{g}_{r}\left[\left[u^{-1}\right]\right]+\sum_{r=0}^{k-1} u^{-1} \mathfrak{g}_{r}\left[\left[u^{-1}\right]\right]+u^{-2} \mathfrak{g}_{k} .
\end{gathered}
$$

Then we have an isomorphism

$$
\bar{\phi}: \frac{\mathbb{O}_{\alpha} \cap \mathfrak{g}\left[u, u^{-1}\right] \oplus \mathfrak{g}}{\left(\mathbb{O}_{\alpha} \cap \mathfrak{g}\left[u, u^{-1}\right] \oplus \mathfrak{g}\right)^{\perp}} \longrightarrow\left(\mathfrak{g}_{k}+\mathfrak{g}_{0}+\mathfrak{g}_{-k}\right) \oplus \mathfrak{g}
$$

such that for the equivalence class of a pair $(f, x) \in \mathbb{O}_{\alpha} \cap \mathfrak{g}\left[u, u^{-1}\right] \oplus \mathfrak{g}$, $\bar{\phi}(f, x)=\left(a_{0}+b_{0}+c_{0}, x\right)$, if $f=u^{-1}\left(a_{0}+a_{1} u^{-1}+\ldots\right)+\left(b_{0}+b_{1} u^{-1}+\ldots\right)+$ $u\left(c_{0}+c_{1} u^{-1}+\ldots\right)+\ldots, a_{i} \in \mathfrak{g}_{k}, b_{i} \in \mathfrak{g}_{0}, c_{i} \in \mathfrak{g}_{-k}, x \in \mathfrak{g}$. The image of $\left(\mathbb{O}_{\alpha} \oplus \mathfrak{g}\right) \cap \mathfrak{g}[u]$ is eaxctly $\Delta_{\alpha, \alpha_{\max }}$.

Remark 3.6. With a different formulation, this result also appeared in [4], where the so-called quasi-trigonometric solutions of the CYBE were classified.

Remark 3.7. We also note the analogy between this theorem and Theorem 2.8. The corresponding Lagrangian subalgebras can be described using the data given in Remark 2.9. 


\section{Lie Bialgebra struCtures on $\mathfrak{g}[u]$ In CASE III}

Finally, let us treat case III, where the associated double is $\mathfrak{g}((u)) \oplus \mathfrak{g}[\varepsilon]$, with the nondegenerate bilinear form given by the formula

$$
\begin{gathered}
Q_{a(u)}\left(f_{1}(u)+x_{2}+\varepsilon x_{3}, f_{2}(u)+y_{2}+\varepsilon y_{3}\right)=\operatorname{Res}_{u=0}\left(u^{-2} a(u) K\left(f_{1}(u), f_{2}(u)\right)-\right. \\
-K\left(x_{3}, y_{2}\right)-K\left(x_{2}, y_{3}\right),
\end{gathered}
$$

for any $f_{1}(u), f_{2}(u) \in \mathfrak{g}((u))$ and $x_{2}, x_{3}, y_{2}, y_{3} \in \mathfrak{g}$. According to [3], the following result holds:

Proposition 4.1. There exists a one-to-one correspondence between Lie bialgebra structures $\delta$ on $\mathfrak{g}[u]$ satisfying $D_{\bar{\delta}}(\mathfrak{g}[[u]])=\mathfrak{g}((u)) \oplus \mathfrak{g}[\varepsilon]$ and bounded Lagrangian subalgebras $W$ of $\mathfrak{g}((u)) \oplus \mathfrak{g}[\varepsilon]$, with respect to the nondegenerate bilinear form $Q_{a(u)}$, and transversal to $\mathfrak{g}[[u]]$.

Recall that any $\sigma(u) \in \operatorname{Ad}(\mathfrak{g}[u])$ induces an automorphism $\sigma(0) \in \operatorname{Ad}(\mathfrak{g})$, which in turn gives an well-defined automorphism $\bar{\sigma}(0)$ of $\mathfrak{g}[\varepsilon]$ via $\bar{\sigma}(0)(x+$ $\varepsilon y)=\sigma(0)(x)+\varepsilon \sigma(0)(y)$. Then $\tilde{\sigma}(u)=\sigma(u) \oplus \bar{\sigma}(0)$ is an automorphism of $\mathfrak{g}((u)) \oplus \mathfrak{g}[\varepsilon]$.

Proposition 4.2. [5] Suppose that $W$ is a bounded Lagrangian subalgebra of $\mathfrak{g}((u)) \oplus \mathfrak{g}[\varepsilon]$, with respect to $Q_{a(u)}$ and transversal to $\mathfrak{g}[[u]]$. Then there exists $\sigma \in \operatorname{Ad}_{\mathbb{C}[u]}(\mathfrak{g}[u])$ such that $\tilde{\sigma}(u)(W) \subseteq\left(\mathbb{O}_{\alpha} \cap \mathfrak{g}\left[u, u^{-1}\right]\right) \oplus \mathfrak{g}[\varepsilon]$, where $\alpha$ is either a simple root or $-\alpha_{\max }$.

The case $-\alpha_{\max }$ has already been analysed in [5]. For an arbitrary simple root we have:

Theorem 4.3. Suppose $a(u)=1$ and let $\alpha$ be a simple root and $k$ its coefficient in the decomposition of $\alpha_{\max }$.

(i) If $k=1$, there exists a one-to-one correspondence between Lagrangian subalgebras $W$ of $\mathfrak{g}((u)) \oplus \mathfrak{g}[\varepsilon]$, with respect to $Q_{a(u)}$, which are transversal to $\mathfrak{g}[[u]]$ and satisfy $W \subseteq\left(\mathbb{O}_{\alpha} \cap \mathfrak{g}\left[u, u^{-1}\right]\right) \oplus \mathfrak{g}[\varepsilon]$ and Lagrangian subalgebras in $\mathfrak{g}[\varepsilon]$, with respect to $\bar{Q}_{\varepsilon}$, transversal to $P_{\alpha}^{-}+\varepsilon\left(P_{\alpha}^{-}\right)^{\perp}$.

(ii) If $k>1$ there are no Lagrangian subalgebras $W$ of $\mathfrak{g}((u)) \oplus \mathfrak{g}[\varepsilon]$, with respect to $Q_{a(u)}$, which are transversal to $\mathfrak{g}[[u]]$ and satisfy $W \subseteq\left(\mathbb{O}_{\alpha} \cap\right.$ $\left.\mathfrak{g}\left[u, u^{-1}\right]\right) \oplus \mathfrak{g}[\varepsilon]$.

Proof. (ii) was proved in [3. (i) If $k=1, \mathbb{O}_{\alpha} \cap \mathfrak{g}\left[u, u^{-1}\right]=u^{-1} \mathfrak{g}_{1}\left[u^{-1}\right]+$ $\mathfrak{g}_{0}\left[u^{-1}\right]+u \mathfrak{g}_{-1}\left[u^{-1}\right]$ and $\left(\mathbb{O}_{\alpha} \cap \mathfrak{g}\left[u, u^{-1}\right] \oplus \mathfrak{g}[\varepsilon]\right)^{\perp}=\mathbb{O}_{\alpha} \cap \mathfrak{g}\left[u, u^{-1}\right]$. Then $\frac{\mathbb{O}_{\alpha} \cap \mathfrak{g}\left[u, u^{-1}\right] \oplus \mathfrak{g}[\varepsilon]}{\left(\mathbb{O}_{\alpha} \cap \mathfrak{g}\left[u, u^{-1}\right] \oplus \mathfrak{g}[\varepsilon]\right)^{\perp}}$ is obviously isomorphic to $\mathfrak{g}[\varepsilon]$. The image of $\left(\mathbb{O}_{\alpha} \oplus \mathfrak{g}[\varepsilon]\right) \cap$ $\mathfrak{g}[u]$ via this isomorphism is $P_{\alpha}^{-}+\varepsilon\left(P_{\alpha}^{-}\right)^{\perp}$. The conclusion follows immediately.

Remark 4.4. This result was also obtained in [7], where quasi-rational $r$ matrices were studied.

Remark 4.5. We observe that the above theorem is analogous to Theorem 2.6 and Remark 2.7 also holds. 


\section{REFERENCES}

[1] Belavin, A., Drinfeld, V.: Triangle equation and simple Lie algebras. Soviet Sci. Reviews, Section C4, 93-165 (1984)

[2] Khoroshkin, S., Pop, I., Samsonov, M., Stolin, A., Tolstoy, V.: On some Lie bialgebra structures on polynomial algebras and their quantization. Commun. Math. Phys. 282(3), 625-662 (2008)

[3] Montaner, F., Stolin, A., Zelmanov, E.: Classification of Lie bialgebras over current algebras. ArXiv math. QA/1001.4824v1

[4] Pop, I., Stolin, A.: Lagrangian subalgebras and quasi-trigonometric $r$-matrices. Lett. Math. Phys. 85(2-3), 249-262 (2008)

[5] Pop, I., Yermolova-Magnusson J.: New $r$-matrices for Lie bialgebra structures over polynomials. To appear in Lett. Math. Phys. ArXiv math. QA/0910.4286v2.

[6] Stolin, A.: On rational solutions of Yang-Baxter equations. Maximal orders in loop algebra. Commun. Math. Phys. 141, 533-548 (1991)

[7] Stolin, A., Yermolova-Magnusson, J.: The 4th structure. Czech. J. Phys. 56(10/11), 1293-1297 (2006)

Department of Mathematical Sciences, University of Gothenburg, Sweden. EMAIL: IULIA@CHALMERS.SE; MD1JM@CHALMERS.SE 